\title{
Diffusione dell'immagine turistica dei laghi della regione prealpina transfrontaliera.
}

\section{Un argomento di interesse particolare alla luce di recenti disposizioni di legge}

Il 17 maggio 1983 è stata approvata dal Parlamento italiano la «Legge quadro per il turismo ed interventi per il potenziamento e la qualificazione e l'offerta turistica». In base a questo provvedimento le varie regioni hanno deliberato allo scopo di dare un nuovo impulso al settore anche tramite il riordinamento dell'amministrazione periferica del turismo. Sia la Lombardia che il Piemonte hanno costituito, con leggi regionali, Aziende di Promozione Turistica il cui scopo è quello di sostituirsi agli Enti Provinciali del Turismo e alle Aziende Autonome di soggiorno per una più efficace opera di sviluppo di un settore che, nella società post-industriale, assume un rilievo sempre maggiore per la creazione di ricchezza e posti di lavoro. Il Canton Ticino, dal canto suo, ottemperando alla legge federale di pianificazione del territorio del 1980, ha elaborato strumenti di piano in cui, vista la propria collocazione strategica nei confronti delle aree transalpine (in particolare la Svizzera interna e la Repubblica Federale Tedesca) e le potenzialità offerte dal proprio territorio, il turismo assume un rilievo notevole (BRUSA, 1987).

Ci si augura da più parti che gli effetti dei provvedimenti legislativi e l'azione degli operatori pubblici e privati sia coronata da successo in una zona interessata da una progressiva perdita di importanza dell'industria come è quella dei laghi prealpini occidentali (Cusio, Verbano, Ceresio e Lario) oggetto di queste riflessioni. La medesima però, pur vantando una tradizione tra le più ricche e nobili, soffre, in particolare dal secondo dopoguerra, i colpi di una concorrenza particolarmente vivace portata soprattutto dalle regioni marine sia italiane che straniere. Le ormai ben note quattro S: sun, sea, sand, sex, sono diventate un simbolo più accattivante per il turista moderno delle immagini più tradizionali e nobili, ma spesso anche troppo oleografiche quando non sbiadite, purtroppo anche dal degrado ambientale, delle regioni lacuali. Inoltre, in questi ultimi anni, anche solo considerando la situazione italiana, la diffusione delle immagini del turismo marino è stata particolarmente curata e abilmente differenziata (BRUSA, 1985), migliorando la propria capacità di coinvolgimento della clientela.
Il problema dell'immagine è ben noto anche al legislatore italiano. Nel testo approvato dalla regione Lombardia il 30 luglio 1986 emerge che i nuovi enti di coordinamento del settore turistico (le Aziende di Promozione Turistica) hanno innanzi tutto il compito della «valorizzazione del patrimonio turistico, paesaggistico, artistico e storico delle località ricadenti nell'ambito territoriale di propria competenza» tramite la propaganda e la pubblicità del medesimo effettuata soprattutto redigendo e diffondendo «materiale illustrativo ed informativo» idoneo allo scopo.(1)

Anche il Presidente dell'Azienda di Promozione Turistica di Varese, nel programma inaugurale dell'attività dell'ente, ha ampiamente ripreso il tema sia in rapporto alla concorrenza internazionale, sia in rapporto a quella attiva nel nostro paese. La necessità di operare su livelli qualitativamente migliori del passato, più articolati e adeguati alle mode in continua evoluzione è un obiettivo dell'azienda varesina che vuole superare gli stereotipi spesso obsoleti quando non negativi, incombenti sulla zona (REDAELLI, 1987).

Nei territori comasco, novarese e varesino le Aziende turistiche hanno i medesimi obiettivi di valorizzazione della regione lacuale che, in larga misura, coincidono con quelli ticinesi. Sarebbe quanto mai necessaria una collaborazione sia per potenziare la portata delle iniziative che per allargare l'offerta di proposte all'esigente turista dei nostri giorni. Qualche cosa si sta muovendo con accordi intervenuti tra Como, Lugano e Varese, ma molto resta ancora da fare.

La regione in esame non ha diffuso recentemente un'immagine integrata di sè pur essendo stata valorizzata unitaziamente all'inizio del secolo - il suo momento d'oro - da una fitta rete di infrastrutture

(1) V. art. 7 comma 2, lett. a, e, b, legge 30.7. 1986, n. 28 della Regione Lombardia.

Carlo Brusa, Prof., Dr, Istituto di Scienze Geografiche, Università di Parma, Borgo Carissimi 10, I-43100 Parma. 
geografiche di circolazione su acqua e su strada ferrata con finalità prettamente turistica (BRUSA, 1979 specie alle pp. 121-162). Oltre a motivi di campanilismo e concorrenza ciò può essere spiegato con l'appartenenza a tre grandi unità amministrative diverse (per non parlare delle minori): le regioni Lombardia, Piemonte e il Canton Ticino. In Italia, inoltre, è suddivisa in tre province: Como, Varese e Novara, mentre in Svizzera gravita su due realtà urbane come Locarno e Lugano che la barriera del Monte Ceneri non ha separato - e per tradizione solo dal punto di vista fisico.

Come si vede è un problema di permeabilità di confini politici, amministrativi e culturali che dovrebbe essere risolto (LEIMGRUBER, 1987) per consentire l'integrazione, suggerita anche dall'ambiente naturale, a vantaggio dell'economia turistica.

\section{«Miti» comuni e strategie diverse nella valorizzazione dell'immagine della zona.}

Limmagine diffusa attualmente dagli operatori pubblici $o$, anche, dai privati non coglie quasi mai l'intero territorio, ma parte dalla meta "puntiforme» o dalla subregione da "valorizzare» e, quasi sempre, si ferma solo a quella e al suo intorno immediato. E tuttavia opportuno verificare, in un ambiente naturalisticamente simile e abbastanza ristretto, se le diverse agenzie di diffusione dell'immagine hanno seguito strategie omogenee o eterogenee per la valorizzazione delle attrattive turistiche, creando eventualmente anche differenzenziazioni di immagine più o meno adeguate alle attuali aspettative del turista. Ciò giustificherebbe, almeno in parte, questa «frammentazione» dell'immagine regionale.

L'analisi riguarda i dépliants pubblicitari proposti dagli operatori pubblici comaschi (principalmente interessati al bacino lariano), da quelli ticinesi (impegnati soprattutto alla promozione del Ceresio e del Locarnese), da quelli novaresi (più attenti alla sponda «grassa» del lago Maggiore e al lago d'Orta) e da quelli varesini (che si affacciano sulla sponda «magra» del Verbano come anche su quella turisticamente meno rilevante del Ceresio). Una strategia comune, rivolta prevalentemente ai turisti mitteleuropei in cerca di caldo sulle coste mediterranee, riguarda la valorizzazione del clima e del paesaggio. Si enfatizza quasi sempre l'aspetto legato al richiamo marino del sole piuttosto che parlare dell'influenza mitigatrice dei bacini lacustri sul medesimo. L'immagine proposta è quella della «mediterraneità» con le sue tipiche essenze vegetali e quella dell'esotismo contenuto nei giardini delle ville.

Così è per il Nord del Lago Maggiore di cui parla un dépliant dell'Ente Provinciale del Turismo diVarese, ma lo è ancor di più per le proposte delle Aziende Autonome di Soggiorno del Lario. A Bellagio si parla di "vegetazione mediterranea», per Cadenabbia si usa la definizione di «riviera delle azalee», per la Tremezzina di «essenze mediterranee» e si calca ancor di più la tonalità con il richiamo al "paesaggio orientale». Sul medesimo tema ritorna un dépliant dell'Ente Provinciale comasco delTurismo che parla di «flora mediterranea». Lo stesso stereotipo è proposto sia dall'Ente di Novara - per i suoi due laghi principali di cui vanta la «fioritura mediterranea e tropicale» che dura tutto l'anno - sia dalla pubblicità delle isole Borromee. La caratterizzazione è ancora più "centrale» per il Canton Ticino che appartiene a quella che i dépliants - specialmente in tedesco e francese - definiscono "Svizzera meridionale», con evidente riferimento alla clientela transalpina.

A Locarno si riprende il «mito» del Sud, parlando di «clima meridionale», di «vegetazione mediterranea» e si propone, diversamente che oltre frontiera, anche in rapporto alla concorrenza marina, la «vita di spiaggia», ricordando pure la presenza di piscine in cui - e questo vale per tutti i laghi in esame - è più consigliabile praticare la balneazione vista la contaminazione delle acque del lago. Lugano, in questa ottica esotizzante, è addirittura paragonata a Rio de Janeiro con riferimento ai due rilievi che dominano il suo golfo: il San Salvatore e il Bré. Tutto questo sforzo di «mitizzazione» della «mediterraneità» si giustifica con la diffusa ansia di attrarre il cliente «nordico» sui laghi in concorrenza con gli operatori del turismo marittimo pure assai attivi nei confronti di un mercato notoriamente ricco. Forse però le immagini e le forzature sono eccessive tenuto conto che il fruitore, ormai, anche grazie al mezzo proprio, può agevolmente raggiungere la vera regione mediterranea.

Presenta una maggiore aderenza alla realtà $e$, probabilmente, assumerà, soprattutto in futuro, una indubbia, se non maggiore, efficacia chi, più precisamente, parla di soleggiamento, dimostrando che Locarno gode della più lunga durata media annua di ore di sole della Svizzera e offre incentivi a chiunque per recarvisi in ogni stagione dell'anno e non solo in quella più calda. Più efficaci - perchè più verificabili in concreto - sembrano però essere le sottolineature dei vantaggi alla salute che la mitezza e, soprattutto, l'effetto tranquillizzante del clima lacustre portano ai villeggianti. Questo è un tema ben sfruttato soprattutto dagli operatori lariani e da quelli del luganese che associano "vacanza» a "cura», fornendo dati precisi sul clima della regione. Non è detto che questo dispiaccia alla clientela transalpina oltre che agli abitanti della vicina "regione città» milanese, tradizionali frequentatori del lago di memoria manzoniana, ben sfruttata - anche con la scelta di nomi di alberghi e ristoranti come "Don Abbondio», il 
«Nibbio», il «Griso», ecc. - sul ramo lecchese del Lario. Non mancano pure itinerari che portano ai luoghi di Renzo, Lucia e dell'Innominato.

Pur non parlando esplicitamente di vantaggi per la salute, anche gli operatori del Cusio vantano un paesaggio «verdeggiante», «dolce»e «tranquillo». Sapendo che ciò non attira molto la clientela giovane (PERUSSIA, 1985, p. 95) si cerca anche di rinnovare l'immagine classica dei laghi che è fatta anche di incantevoli panorami e di belvedere presenti quasi ovunque.

Lugano viene così definita città di «cultura» (non si dimentichi oltre all'attività congressuale, e l'assoluto rilievo mondiale della Pinacoteca Thyssen Bornemisza), città da «godere» e città di «divertimento». Per Locarno si vanta sia la possibilità di praticare vari sport - non ultimi, nelle vicinanze, quelli sulla neve - sia quella del «divertimento serale» in locali di classe come il Kursaal.

Il richiamo agli ambienti circostanti in cui si può praticare soprattutto lo sci che attrae una clientela giovanile è ricorrente anche per il Pian dei Resinelli e il Piano d'Ernia sopra Lecco dove si scia «con il lago a un tiro di schioppo», per le alture sopra Locarno, per il Mottarone tra il lago Orta e Stresa, per la Val d'Intelvi, prossima a Como e alla Svizzera, e assai vicina a Milano tanto da favorire la pratica dello sport preferito anche a chi dispone di poco tempo da perdere per il viaggio.

Più tradizionale e classico è il richiamo all'ambiente selvaggio delle vallate che si irradiano dai laghi e che sono in stretta simbiosi con essi sia per vicende economiche che storico-culturali, oltre che per fortune turistiche (BUZZETTI, 1976, pp. 220-221). Il più efficace intervento, anche per l'abilità degli accostamenti sia iconografici che scritti, è quello a favore del retroterra Locarnese servito dall'unica ferrovia turistica ancora operante nella zona, dopo la soppressione delle linee Menaggio-Porlezza e Ponte Tresa-Luino (EvANGELISTA, 1986) oltre che di quelle delle valli varesine: la Domodossola-Locarno. Di questa si vanta - in concorrenza con l'automezzo che è in difficoltà su un percorso accidentato di montagna - anche la «sicurezza e la comodità» che consentono una "percezione completa» del paesaggio «verde» e dal carattere «selvaggio»e ancora largamente preindustriale. La ferrovia, inoltre, congiungendo gli assi del Gottardo e del Sempione è il "più rapido collegamento" tra il Ticino e il Vallese.

Dai retroterra di Menaggio e di Bellagio, alla regione Lecchese con la ben nota Valsassina, a Stresa con il Mottarone e alle valli Varesine viene un invito a visitare un ambiente assai diverso da quello costiero lacuale pur così vicino.

In molti casi, però, si parla solo di differenze climatiche, di «verde», ma in altri, soprattutto elvetici, si propone la conoscenza della cultura materiale pre- sente sia nelle antiche tipologie insediative che negli ormai numerosi musei delle Valli Ticinesi: preziose raccolte che testimoniano la «ricchezza e la vivacità del mondo alpino» di cui si suggerisce una riscoperta e una maggiore attenzione all' «anima popolare». Nel Varesotto, invece, si invita la gente a particolari esposizioni e a manifestazioni fieristiche tra cui, efficacemente pubblicizzata, è quella mensile di antiquariato di Castiglione Olona che, a ricordo anche della storia e dell'arte locali, prende nome dal suo più noto antico cittadino: il cardinale Branda Castiglioni.

Tutto ciò suggerisce un rinnovamento dell'uso turistico tradizionale della regione e ne allarga la fruizione (CORNA PELLEGRINI, 1985, p. 3; PERUSSIA, 1985). Questo avviene anche con proposte, prevalentemente elvetiche, di itinerari a piedi. Sono rivolte sia all'ospite delle vicine stazioni lacuali sia a chi, in giornata, si può spostare dalle non lontane aree urbanizzate a una regione «incantevole per vacanza e cura» come sottolinea la pubblicità dell'ente turistico Valli di Lugano. Le mete possono riguardare sia aspetti scenografici del paesaggio, sia particolari realtà insediative, sia, come nel caso del Monte San Giorgio, fra due rami del Lago di Lugano, l'ambiente vegetale, faunistico e, soprattutto, paleontologico.

Gli operatori luganesi sono attenti sia al «vicino» sia al «lontano» e si propongono ai loro ospiti mitteleuropei, come testa di ponte verso l'Italia, sia alla scala della «regione dei laghi» con itinerari per Stresa e le Isole Borromee o Como, sia verso Milano sia - ancor più lontano - verso mete che danno "prestigio» - anche se si può dire solo che ci si è stati in una giornata - come Venezia e Firenze. Una speciale attenzione è rivolta ad un tipo di clientela italiano particolarmente qualificato dal punto di vista del reddito cui Lugano propone un "pacchetto» di quattro giorni di vacanza sul lago, nei migliori hotel, con trasporto aereo da e per Roma e possibili serate anche al vicino Casino di Campione.

Questo centro, exclave italiano in terra svizzera (PEDRESCHI, 1964), anche dal punto di vista della nuova legge lombarda di promozione del turismo, come Livigno, fa a sè. La sua «clientela», infatti, è ben più selezionata economicamente di quella normale della regione e la sua immagine si collega anche a manifestazioni prettamente mondane di portata internazionale oltre che, come ovvio, al gioco d'azzardo.

La posizione dei centri rispetto alle aree da cui proviene il turista è pure particolarmente ben evidenziata nei dépliants. Nei testi ad es., Varese è definita un "carrefour», Ponte Tresa è un valico che con Zenna e Gaggiolo consente di evitare le code a chi si sposta da Nord a Sud lungo la via del Gottardo, Stresa e la regione lacuale novarese, sono associate al Sempione. 
La collocazione regionale è evidenziata ancor meglio nelle carte da cui si deduce sia l'area di residenza della clientela potenziale a cui pensano di rivolgersi gli operatori, sia quella parte di regione dei laghi che intendono "valorizzare». Quest'ultima è impreziosita, a cura degli enti turistici, da illustrazioni a volo d'uccello che evidenziano ampie zone del Cusio e del Verbano, del bacino Superiore del lago Maggiore con Locarno, del lago di Lugano o di quello di Como. Non manca neppure qualche visione di tutto il territorio con sempre ben presente lo sfondo delle montagne alpine e l'indicazione del nome delle vette principali. Nella cartografia della regione occorre inoltre notare che, tra i laghi principali, il Cusio scompare quasi sempre sia per la sua posizione all'estremo lembo occidentale dell'area, sia per il minor rilievo turistico nel sistema complessivo. L'attenzione si focalizza sul Nord per le carte e dépliants stampati sia al di qua che al di là del confine italo-svizzero. Mentre verso l'Europa transalpina - soprattutto tramite l'evidenziazione dei valichi più vicini: il Sempione per il Novarese, lo Spluga per il Comasco, il S. Bernardino e il Gottardo per il Ticino e il Varesotto - si arriva tranquillamente alla regione germanica a sud non si scende sotto Milano e a est e a ovest, solo sporadicamente, si evidenziano Venezia per interessi turistici e, soprattutto nel Novarese, Genova e Torino per la maggior vicinanza al Verbano. Il tracciato del confine, inoltre, non è sempre evidenziato a testimonianza della sua permeabilità e apertura ai flussi turistici e della sua inesistenza, come ostacolo, agli spostamenti di vacanza.

\section{Considerazioni conclusive}

L'immagine offerta dalla pubblicità turistica enfatizza, come da tradizione, gli aspetti naturalistici. Le osservazioni più classiche e ricorrenti sono integrate da proposte nuove soprattutto nel Canton Ticino, che ha pure il vantaggio di rivolgersi ad un mercato nazionale potenzialmente più vasto di quello italiano il quale soffre da anni della concorrenza più ravvicinata delle stazioni marine. La regione varesina che già vanta minor capacità di richiamo di quelle svizzera, comasca e novarese deve, più delle altre, sforzarsi di migliorare la propria immagine e di meglio caratterizzarla. Questo dato non emerge solo dall'analisi dei dépliants ma da approfondite e recenti ricerche sulla percezione di chi fa turismo nella zona (PERUSSIA, 1985). L'antico ruolo nodale di Varese - che si ritrova all'incrocio delle vie per il Verbano, il Lario ed il Ceresio - andrebbe ulteriormente potenziato. In particolare questo dovrebbe essere fatto anche nei confronti della clientela commerciale che si servirà sempre di più dell'aeroporto della Malpensa, in fase imminente di ampliamento, senza dimenticare quella delle vicine aree della «regione-città» milanese. Per queste ultime, anche la diffusione delle moderne istanze ecologiste suggerirebbe l'elaborazione di una serie di proposte più stimolanti e aggiornate di quelle "classiche». Lo stesso turista straniero potrebbe sia soggiornare nel Varesotto a prezzi più convenienti che in Svizzera, sia scegliere di attraversarlo con i suoi valichi alternativi a quelli comaschi ormai congestionati. Qualcosa si sta già muovendo nel creare questo tipo di immagine, ma molto di più potrà essere fatto grazie sia alle disposizioni della recente legge italiana sul turismo, sia alla collaborazione con la realtà ticinese per costituire un sistema territoriale integrato in una regione che, dal punto di vista formale, presenta già caratteristiche unitarie.

\section{Zusammenfassung}

\section{Zur Verbreitung des Vorstellungsbildes \\ der Alpenrandseen in der italienisch-schweizeri- schen Grenzregion}

Der vorliegende Aufsatz entstand unter dem Eindruck eines neuen italienischen Gesetzes zurTourismusförderung. Er untersucht das Vorstellungsbild, das die Ferienprospekte von einem traditionsreichen Erholungsgebiet vermitteln, das allerdings von mehreren politischen und administrativen Grenzen zerschnitten ist. Die häufigsten «Mythen» sind der mittelmeerische Charakter und die natürlichen Elemente des Raumes. Nicht vergessen werden aber auch - dies besonders auf schweizerischer Seite - die Aspekte Sport und Spiel sowie das kulturelle Erbe, die sich als recht effiziente Bestandteile des Bildes erweisen. Aus der Analyse der Prospekte ergibt sich, daß eine bessere Koordination zwischen den verschiedenen Fremdenverkehrsorganisationen wünschenswert wäre. Damit könnte eine bessere Inwertsetzung des touristischen Potentials über das Vorstellungsbild erreicht werden.

\section{The Diffusion of a Touristic Image: the Lakes in the Swiss-Italian Border Area}

The present paper arose from the publication of a new law on the promotion of tourism in Italy. It focuses on the image of the Lakes area in the SwissItalian border region-a traditional tourist area-as conveyed by the various regional tourist agencies through their information brochures and leaflets. The two «myths» appearing most frequently are the mediterranean character and the natural features of the region in question. The Swiss promotors also 
emphasize sports activities and the cultural heritage, two elements of the image which are particulary efficient components of the image. The analysis of the brochures and leaflets reveals that the different tourist agencies ought to coordinate their information activities more fully in order to create an integrated image of the region. This in turn could help to better exploit the tourist potential of the region.

\section{Bibliografia}

BUZZETTI, L. (1976): Industria e turismo nell'area prealpina lacuale. II bacino sud-occidentale lariano. In: SAIBENE C. (a cura di), Ricerche sull'assetto territoriale della Lombardia, Milano, Vol. II, 7-318.

BRUSA, C. (1979): Evoluzione di un'immagine geografica. ॥ Varesotto turistico, Torino.

BRUSA, C. (1985): Images publicitaires des régions du tourisme maritime en Italie. GUERIN Y./GUMUCHIAN H., Les re- présentations en actes, Actes du Colloque de Lacheraines, Grenoble, 231-236.

BRUSA, C. (1987): La recente legge svizzera di pianificazione del territorio e la sua applicazione in Canton Ticino. "Boll. Soc. Geogr. Ital.", XI/IV, 1-20.

CORNA PELLEGRINI, G. (1985): La ricerca geografica e psicologia sul turismo. II caso varesino. In: CORNA PELLEGRINI G./ FRIGERIO A. (a cura di), Turismo come e perchè, Modello di ricerca geografica e psicologica, Varese, 1-9.

EVANGELISTA, G. (1987): Nel centenario della ferrovia dei tre laghi, "Lombardia Nord-Ovest", 58, 61-64.

LEIMGRUBER, W. (1987): II confine e la gente. Interrelazioni spaziali, sociali e politiche nella regione di confine fra la Lombardia e il Canton Ticino, Collana dell'Ist. di Scienze Geogr. Università di Parma, no. 7, Varese.

PEDRESCHI, L. (1965): L'exclave italiano in terra svizzera di Campione d'Italia. In: "Atti del XIX Congr. Geogr. Ital.", Como, Vol. escursioni, 39-52.

PERUSSIA, F. (1985): Aspetti psico-sociali del turismo. In: CORNA PELLEGRINI G./FRIGERIO A. (a cura di), 11-100.

REDAELLI, E. (1987): Prime linee programmatiche per l'attività dell'Azienda di Promozione Turistica del Varesotto, Azienda di Promozione Turistica, Varese. 